\title{
Some data on the influence of structure and chemical composition on the ratio of fracture toughness and tensile brittleness of titanium alloys.
}

\author{
A. Volkov ${ }^{1}$, M. Leder $^{1}$, A. Grebenshchikov ${ }^{1}$, N. Volkova ${ }^{1}$, E. Shushakova ${ }^{1}$ \\ ${ }^{1}$ PSC VSMPO-AVISMA Corporation, Parkovaya st. 1, Verkhnaya Salda 624760, Russia \\ Email*, moleder@vsmpo-avisma.ru
}

\section{Introduction}

The development of the aerospace industry is also associated with the development of a complex of mechanical properties of titanium alloys. The main high-strength pseudo-beta titanium alloys were developed in the $70 \mathrm{~s}-80 \mathrm{~s}$,but at the current moment they have not occupied a $\mathrm{m}$ ainsegment in applications, significantly inferior in terms of the volume to the Ti6Al4V alloy. We can formulate the main requests from the designers, which are relatively constant: reducing the weight of the structure, increasing strength, increasing stiffness, increasing fracture toughness. If an increase in the strength and fracture toughness of a material is possible and is one of the main tasks of the developers of materials, then an increase in the rigidity of titanium alloys is possible only through the use of composite materials. Reducing the weight of the structure within the framework of titanium alloys (the density is specified) is possible only through a decrease in the cross-sectional area of the part, which is inversely proportional to strength, i.e. with increasing strength.

In addition, the increase in strength leads not only to a reduction in the weight of the final part, but also to a reduction in the weight of the stock, which ensures a reduction in cost, which is one of the most actual market demands.

So for parts with a section> 1 "when cooled in air relative to the base strength level of UTS = 1050 MPa of Ti6Al4V alloy, it is possible to increase the strength on the VST55531 alloy to UTS = $1650 \mathrm{MPa}$, which is equivalent to a 1.6 times reduction of the section or $37 \%$. For thin sections with the possibility of quenching in water, the equivalent reduction in the section can be 1.4 times or $28 \%$. limitations.

Thus, an increase in the strength of titanium alloys is a very promising and realiable direction, which, however, has several

With an increase in strength for all classes of titanium alloys, ductility under tension and fracture toughness decrease. Upon reaching the critical strength at which the failure occurs immediately after the elastic deformation, without any plastic deformation. That is plasticity (relative elongation and reduction area) under tension $=0$. The material in this state is a tensile macro brittle. Similarly, at a certain strength, the fracture toughness may decrease to 0 . This state will be called "toughness brittle».

The plasticity realized by the material during plastic deformation depends on the stress-strain state. In order of increasing severe of loading: compression, torsion and tension. Tension can be considered the most severe loading at which the minimum plasticity resource is realized. Therefore, in tensile brittle states, true strength is as close as possible to the test for compression or hardness.

The danger of brittleness lies in the unpredictability of the results of its loading. When the threshold of brittle tensile strength is exceeded, with a further increase in the true strength (determined by hardness), an increase in tensile strength is observed in a small area (Fig. 1). With further increase in true strength, maximum tensile strength decreases. And there is a range of UTS, in which failure can occur with a certain probability. The greater the difference between the true strength and the threshold brittleness strength, the greater the range of possible UTS (Fig. 1).

The dimly lit question remains the ratio of tensile brittle and toughness brittle strengths, as well as the impact on this ratio of chemical composition, structure parameters, crystallographic texture etc. There is little systematic experimental data because the main task is to avoid brittleness states. 


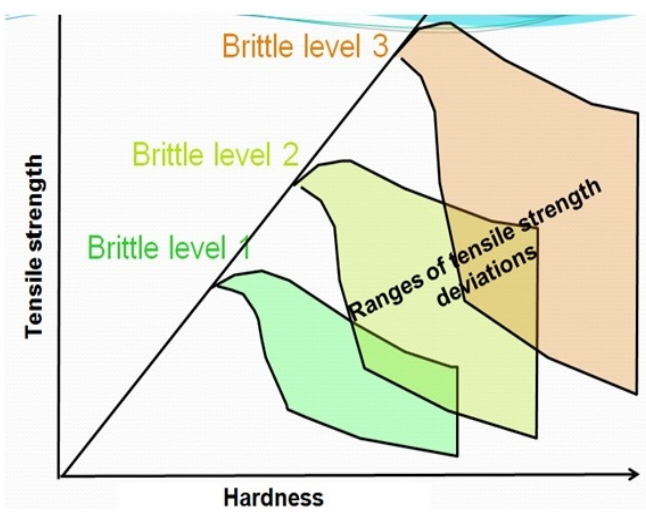

conditions.
Table 1 Chem. composition of the investigated alloys.

\begin{tabular}{|c|c|c|c|c|c|c|c|}
\hline & $\mathrm{Al}$ & $\mathrm{V}$ & $\mathrm{Mo}$ & $\mathrm{Cr}$ & $\mathrm{Fe}$ & $\mathrm{O}$ & $\mathrm{Mo}_{\text {эKB }}$ \\
\hline Exp.1 & 4 & $\mathrm{~V}$ & $\mathrm{~V}$ & $\mathrm{~V}$ & & & 11 \\
\hline VST2 & 5.4 & 3.6 & 1.7 & 0.7 & 0.75 & 0.23 & 7.4 \\
\hline VST3553 & 3.1 & 4.9 & 5.15 & 2.8 & 0.4 & 0.18 & 13.6 \\
\hline VT22 & 5.1 & 4.8 & 4.9 & 0.9 & 0.9 & 0.12 & 12.7 \\
\hline
\end{tabular}

Fig.1 Scheme of the behavior of tensile strength in brittle

\section{Material and experiments}

The work carried out rods deformed in a + b-field, chemical composition is given in table. 1 .

The tensile test was carried out on samples of $\varnothing 6.0 \mathrm{~mm}$ with the gage length 4D according to ASTM E8. The tensile was carried out on an MTS Z100 testing machine with a nominal force of $10 \mathrm{kN}$ at room temperature. Impact toughness was tested on samples with a cross section of $10 \times 11 \mathrm{~mm}$ with a V-shaped notch and a pre-grown fatigue crack. The torsion test was carried out on samples of $\varnothing 10 \mathrm{~mm}$ and a working part length of $50 \mathrm{~mm}$, on a universal testing machine TNS-DW 05 with a nominal torque of $500 \mathrm{~N}^{*} \mathrm{~m}$. The microstructure was investigated on microslises after preparation on a composite wheel and final polishing using Colloidal Silica of $0.05 \mu \mathrm{m}$ dispersion. The microstructure and surface damage was investigated on a FEI Quanta 3D FEG and SEM Zeiss Sigma 300 VP scanning electron microscope with an accelerating voltage of up to $30 \mathrm{kV}$.

\section{Results and discussion}

The work was carried out on alloys, with significant hardening due to precipitation age hardening.

For the experimental alloy of the martensitic class (Table 1), the strength of the aging regime was varied in different initial states. At hi-temperature aging (HTA), minimal hardening was realized due to large precipitations of the secondary phases. At low temperature aging (LTA), the maximum hardening was realized due to the fine discharge of the secondary phases.

During beta quench a "martensitic transformation occurs. Low temperature aging (LTA) results in a very fine mixture of a and $\beta$ phases (Fig. $3 \mathrm{~d}$ ), which supply increase in material strength up to $460 \mathrm{HV}$ leads to tensile brittleness (Fig. 2 a), however, the impact strength does not fall to zero. The coarsening of $\alpha$ and $\beta$ phases after hi-temperature aging (HTA) (Fig. 3 a) reduces hardness to $354 \mathrm{HV}$, and the material is not tensile brittle, i.e. shows significant elongation and RA, also showing a twofold increase in impact toughness (Fig. 2b). The magnitude of microplastic deformation, determined by the local deformation of the edges of the dimples and fracture facets, decreases during the transition to the tensile brittle condition, but does not undergo qualitative changes - remains micro ductile (Fig. 3 a, d). An atypical feature is the grain boundary fracture in the HTA condition, which is less energy-intensive compared to the predominantly intragranular fracture in the tensile brittle LTA condition.

The transition to a globular structure with about $20 \%$ of the primary globular a-phase after $a+\beta$ quench to metastable allows for relatively high ductility (EL up to 12.5 and 19.2\%) with comparable hardness, but significantly lower impact toughness in the corresponding HTA and LTA conditions compared to beta quench condition. Tensile brittle beta quench LTA condition has a significantly higher impact toughness compared to $\alpha+\beta$ quench LTA condition, which indicates that there is no unambiguous relationship between the tensile brittle and level of impact toughness.

The lamellar structure after beta anneal with a close fraction of the primary a-phase and quenching to the metastable bphase reveals the same tendencies as the beta quench condition (with the transformation of $a$ "martensite) (Fig. 2). The magnitude of microplastic deformations in the tensile brittle condition after LTA is not significantly different from microplastic deformation in HTA condition with significant macro-plastic deformation under tension (Fig. 3c, f). The level of impact toughness also does not fall to 0 in the tensile brittle condition after LTA (Fig. 2 b) and corresponds to the corresponding states in the beta quench condition. In general, the classic picture is observed on this experimental alloy - the lamellar structure has a significantly 
lower ductility and a higher fracture toughness compared to the $\alpha+\beta$ structure.

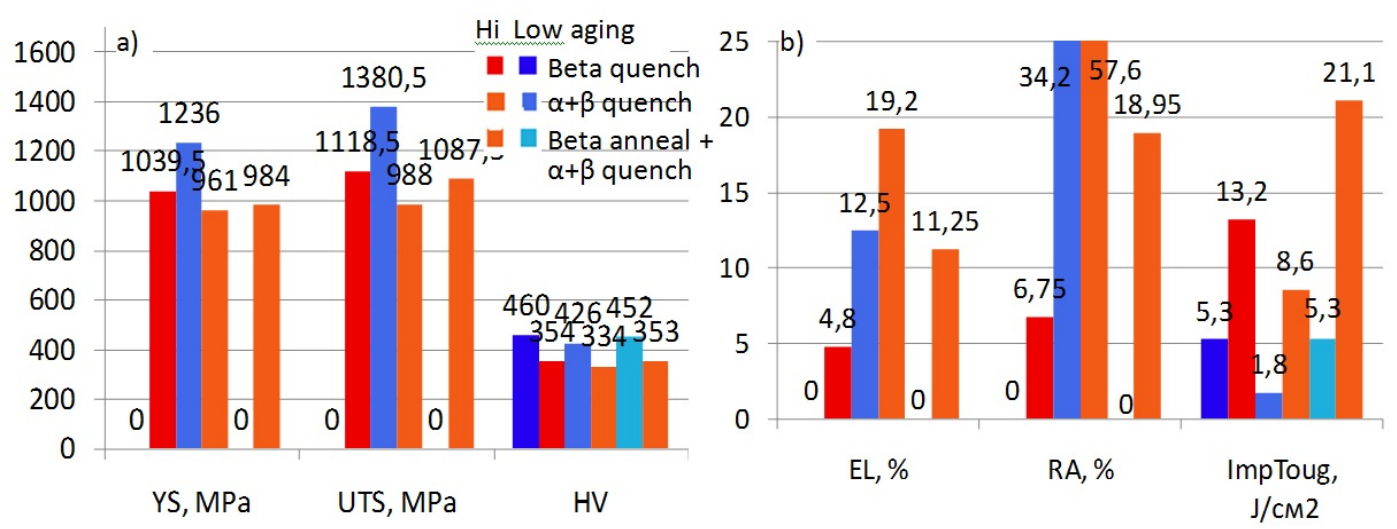

Fig. 2 Mechanical properties of the experimental alloy rod after aging at high and low temperatures with different types of initial structure: beta quench; $a+\beta$ quench; beta anneal $+a+b$ quench.

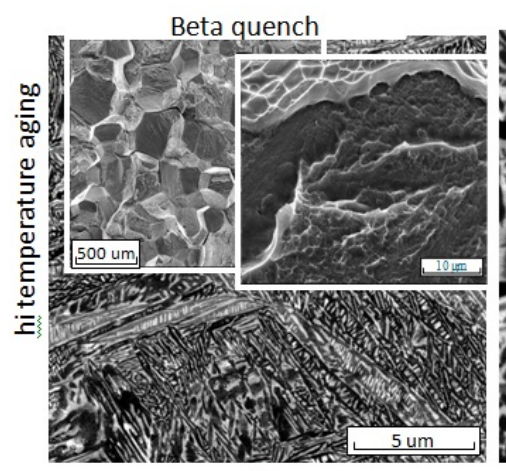

a
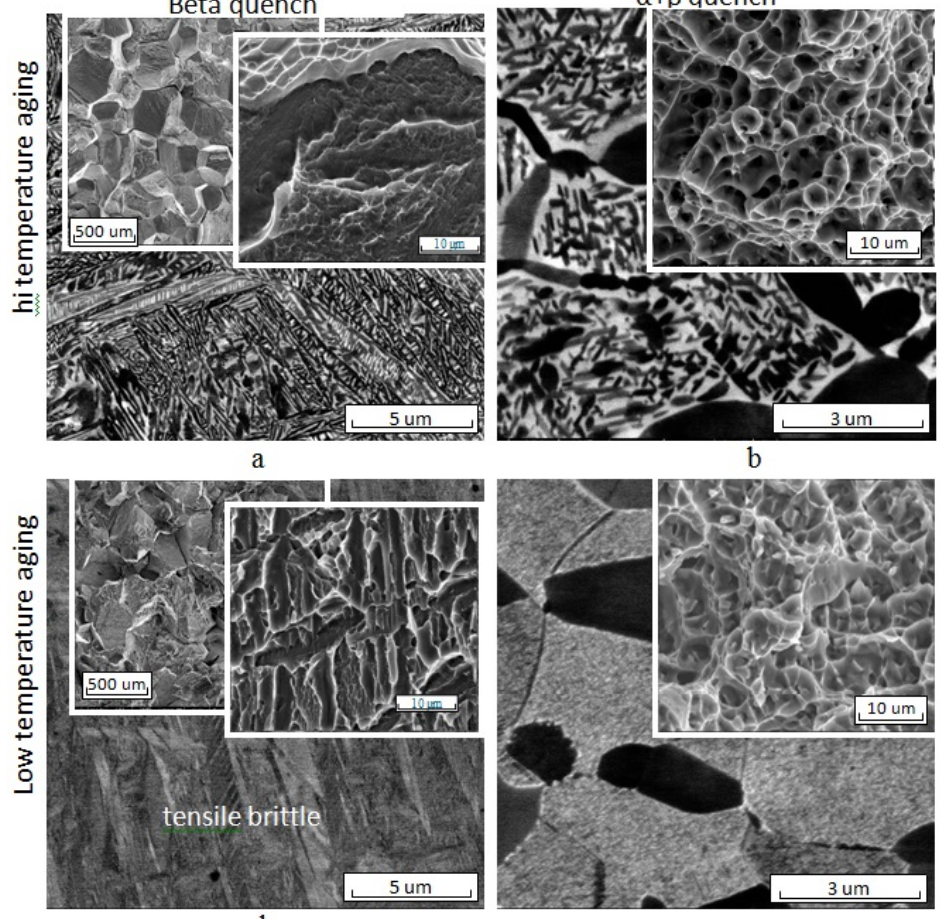

$\mathrm{b}$
Beta anneal $+a+b$ quench

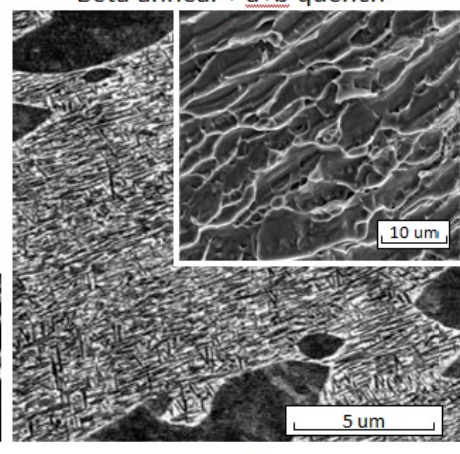

c

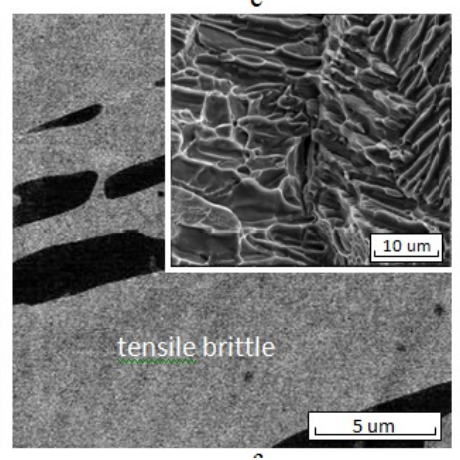

d

e

Fig.3 Microstructure of the experimental alloy rod after aging a, b, c) at high temperature; $d, e, f)$ aging at low temperature, with a different type of initial structure: $a, d)$ beta quench; $b, e) a+\beta$ quench; $c, f)$ beta anneal $+\alpha+\beta$ quench.

For a somewhat less alloying VST2 alloy (Table 1), the quenching temperature varied the proportion of the primary aphase ( $\gamma a 1)$ on the $a+\beta$ processed structure and beta anneal structure (Fig.4.5), followed by thermo strengthening by aging in one mode. All states with a lamellar structure after beta anneal were tensile brittle. All $\alpha+\beta$ processed condition had non-zero elongation and RA (Fig. 4 b). At the quenching temperature of the corresponding $\gamma_{a 1}$, up to $18 \%$ inclusive, the alloy is quenched by a "martensite. With a larger $\gamma_{a 1}$ - on the beta phase.

States with the same $\gamma_{a 1}$ had very close strength, based on hardness data. At the same time, as in the experimental alloy, the difference in the magnitude of the microplastic deformation of the fracture relief is not significantly different between the beta anneal (tensile brittle) and $\alpha+\beta$ processed condition (Fig. $5 \mathrm{a}, \mathrm{d}, \mathrm{e}, \mathrm{f}$ ). At maximum $\gamma a 1=40 \%$, the difference in macroplastic deformation between the $\alpha+\beta$ processed and beta anneal conditions is maximal and significantly larger than the state with $\gamma_{a 1}=$ $6 \%$ (Fig. 4 b), however the difference between the magnitude of microplastic deformation does not increase and remains close. 
When $\gamma a 1=6 \%$, the impact toughness has close values for both initial states (Fig. 4). When $\gamma a 1=18 \%$, the impact toughness in the tensile brittle beta anneal condition is lower than in the $\alpha+\beta$ processed condition, which is atypical. When $\gamma a 1$ $=40 \%$ in the beta anneal condition has a greater impact toughness, despite the tensile brittleness, compared with the $\alpha+\beta$ processed condition.,
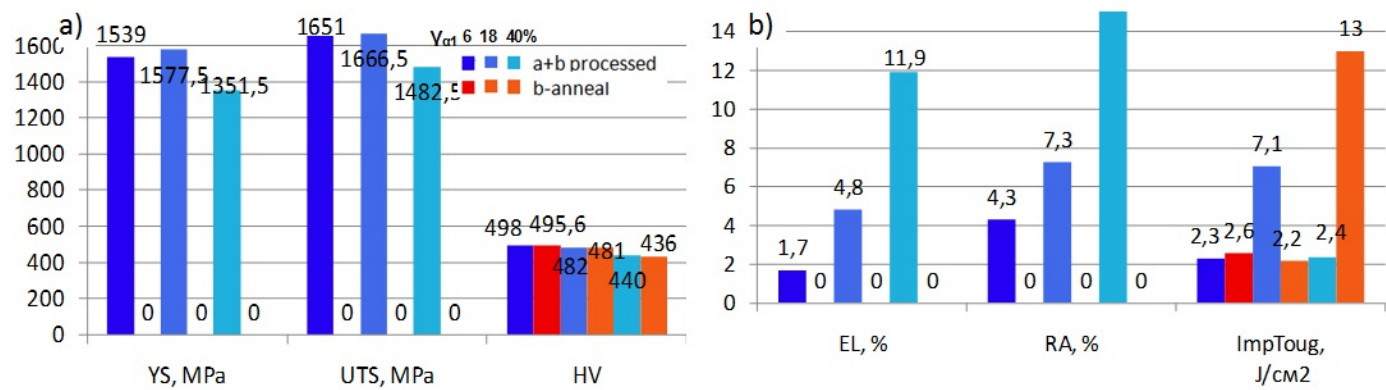

Fig.4 Mechanical properties of a VST2 alloy rod with various portions of the primary a-phase with different initial microstructure: $\alpha+\beta$ and $\beta$-transformed.
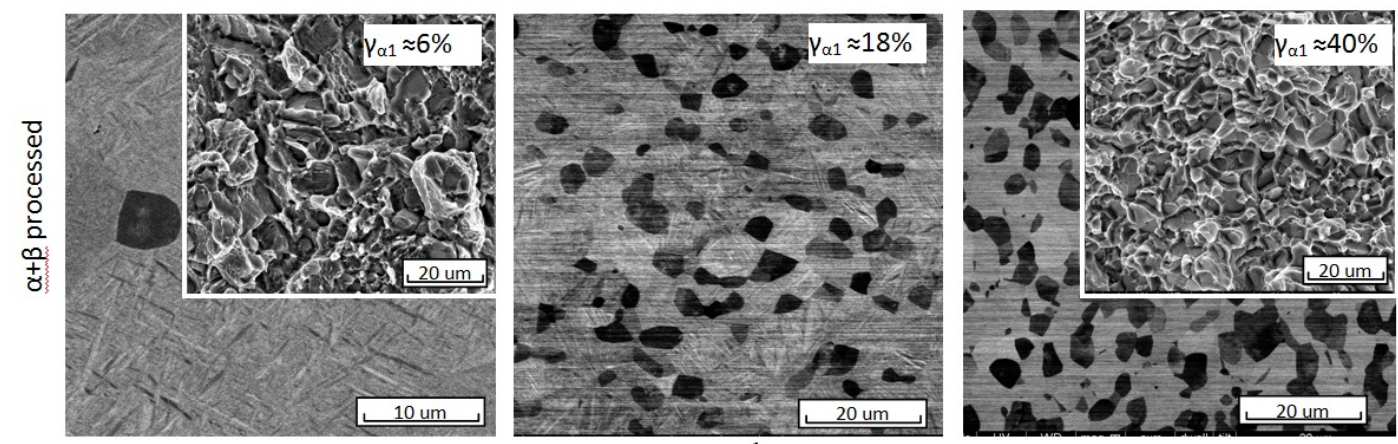

a

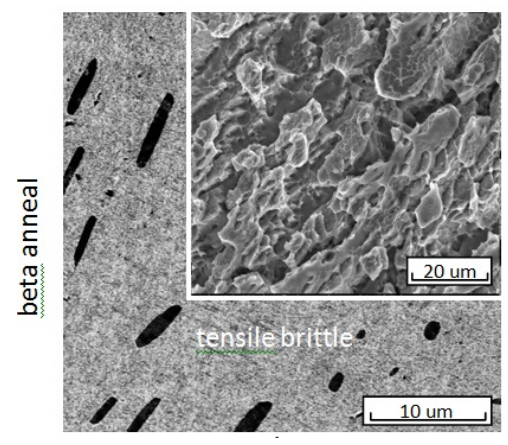

d b

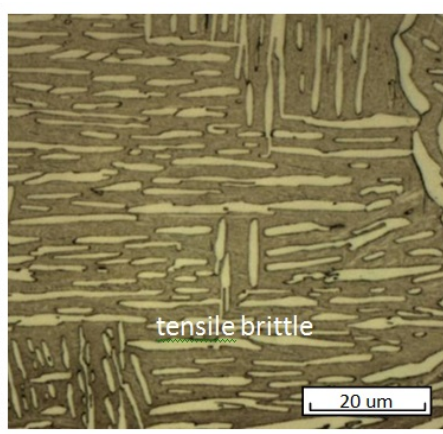

$\mathrm{e}$
C

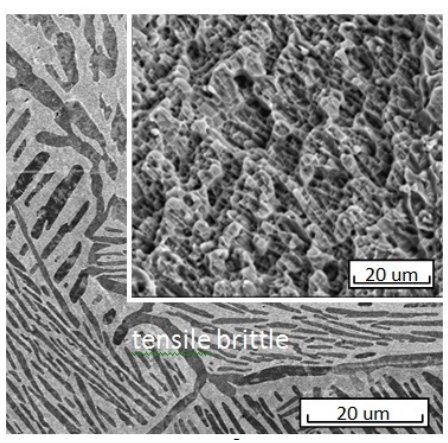

f

Fig. 5 Microstructure and fractograms of the fracture surface of a VST2 alloy rod with a different type of initial structure a, b, c) $a+\beta$ processed; $d, e, f)$ beta anneal, quenching in water for a different fraction of the primary a-phase:a,d) $6 \%$; b,e) $18 \%$;,$f$ ) $40 \%$.

It is difficult to talk about the reasons for the change in the ratio of impact toughness with $\gamma a 1=18 \%$ relative to $\gamma_{a 1}=$ $40 \%$. Most likely the cause is a change in the fraction of the primary a-phase, since the phase composition after quenching for $\gamma_{a 1}$ $=6$ and $18 \%$ does not change, but this also causes a change in the ratio of impact toughness.

This alloy also shows the absence of a direct link between the level of impact toughness values and the transition to tensile brittleness. The level of impact toughness depends in a complex way on the type of structure and the proportion of the primary a-phase.

In the pseudo-beta VT22 alloy, the strength variation was made due to the change in the aging temperature (Fig. 7). LTA and HTA were selected so that the LTA state was tensile brittle, and the HTA was ductile. The difference in the strengths of the LTA and HTA states was maintained at a minimum and was 18-68 HV (80-250 MPa in terms of UTS) (Fig. 6). The ductility of the material was recorded by tensile and torsion tests. The primary a-phase volume fractions for quenched conditions from the $\alpha+\beta$ region was about $12 \%$. 
For $\alpha+\beta$ quench condition impact toughness for LTA and HTA was the same (Fig. 6). When tested for torsion, the LTA state was brittle, and HTA state it showed a slight plasticity, i.e. coincided with tensile brittleness.

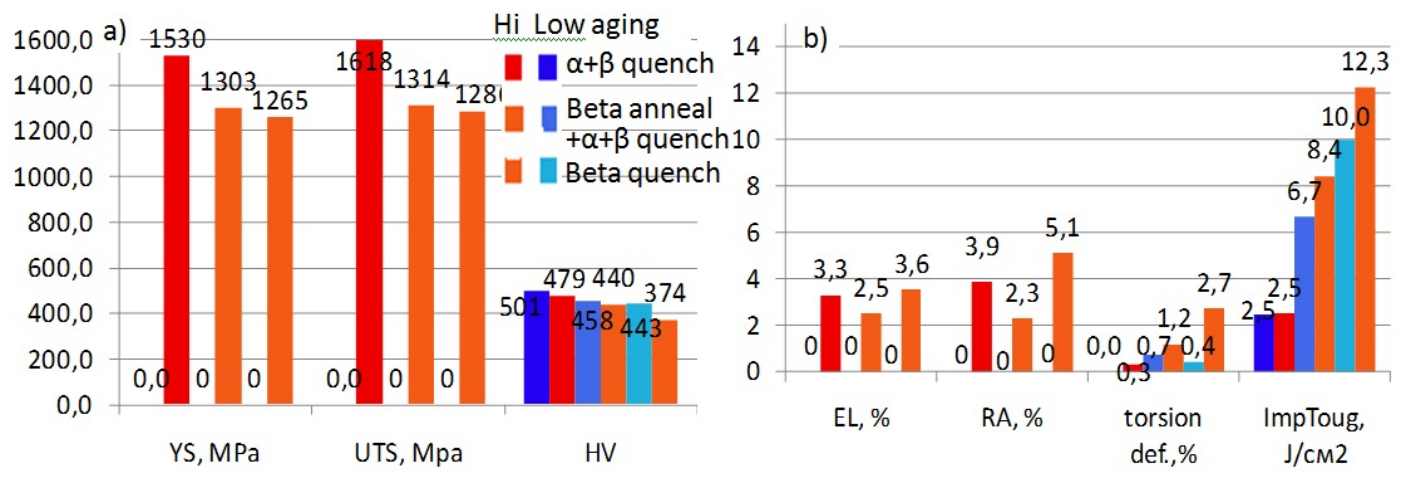

Fig.6 Mechanical properties of the VT22 alloy rod after aging at high and low temperatures with different types of initial structure: $\alpha+\beta$ quench; beta anneal $+\alpha+\beta$ quench; beta quench.
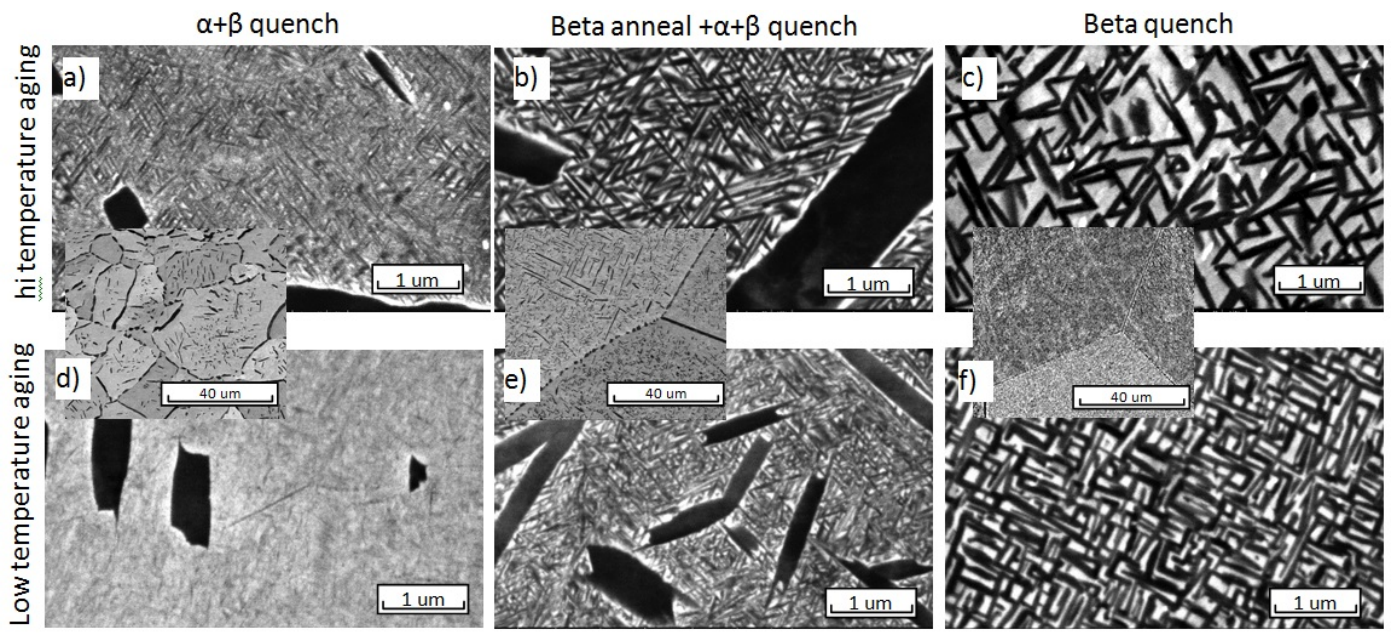

Fig. 7 Microstructure of the VT22 alloy rod after aging a, b, c) at high temperature; $d, e, f)$ aging at low temperature, with a different type of initial structure:a,d) $a+\beta$ quench; $b, e)$ beta anneal $+a+\beta$ quench; $c, f)$ beta quench.

The transition to a lamellar structure using beta anneal leads to an increase in $\beta$-grain up to $180 \mu \mathrm{m}$ (Fig.7) and a decrease in the strength of the transition to tensile brittleness (Fig.6). Impact toughness for LTA is slightly lower than HTA and more than 2.7 times the $\alpha+\beta$ quench condition (Fig. 6). During torsion, the material experiences plastic flow, including in the tensile brittle LTA condition, although the deformation of the torsion LTA is less than the HTA state.

Quenching from the beta region eliminates the primary a-phase and leads to an increase in the $\beta$-grain (Fig. 7). Impact toughness for LTA is slightly lower than HTA and almost 2 times more than beta anneal condition (fig.6). As in the beta anneal condition during torsion, the material get plastic flow, including in the tensile brittle LTA condition, although the torsion deformation of LTA is less than the HTA state.

In general, for the VT22 alloy there is a classic dependence of greater impact toughness and less plasticity for the lamellar structure compared to the globular structure.

The coincidence of the brittle state under tension and torsion for the $\alpha+\beta$ quench condition, as well as the presence of plasticity under torsion in other brittle states, confirms the most stringent conditions from the point of view of the implementation of plastic deformation are realized under tension.

Analyzing the general impact toughness behavior tendencies near the tensile brittle ductile transition on different classes of alloys and microstructures, we can say that the tensile brittle ductile transition is not related to the toughness brittle transition, and that the impact toughness function does not have discontinuities during the brittle ductile transition.

The dependence and behavior of impact toughness at tensile brittleness is complex and depends on a large number of factors, but one of the most significant influense is the type of the primary a-phase structure and its specific fraction. It should be borne in mind that the behavior of impact toughness is very dependent on chemical composition of the alloy and is unlikely to be versification. 
The monotonous behavior of fracture toughness near the tensile brittle ductile transition makes it possible to absolutely safely realize states with maximum strength, i.e. which are as close as possible to the tensile brittle ductile transition. It is sufficient to ensure the plasticity margin for the scatter over the cross section of the stock, and also take into account the provision of the required level of fracture toughness, which will decrease with increasing strength.

\section{Conclusion}

1. Increasing the ductility of alloys with simultaneous consideration of the level of fracture toughness can be a prerequisite for using higher strength states on titanium alloys.

2. Tensile brittleness on titanium alloys is not associated with «toughness brittleness»

3. Fracture toughness behaves monotonously and continuously near the tensile brittle ductile transition.

4. The behavior of fracture toughness near the tensile brittle ductile transition is complex and is caused by a large number of factors, including the type of structure of the primary a-phase and its shares.

5. Tensile brittleness has much less strength than toughness brittleness.

6. The tension stress $\backslash$ strain state in terms of brittleness can be considered the most severe.

7. Beta transformed structures compared to $a+b$ structures has a lower ductility on all alloys. The ratio of fracture toughness end tensile properties depends on the alloy and state.

8 . It is enough to provide in the material $1-2 \%$ elongation to avoid tensile brittleness.

\section{References}

